\title{
Verbesserte Früherkennung von Darmkrebs
}

Der Bundesausschuss der Ärzte-Krankenkassen hat mit Wirkung vom 1. Oktober 2002 eine Änderung der Krebsfrüherkennungsrichtlinien für das kolorektale Karzinom beschlossen. Danach können sich alle Personen zusätzlich zum fäkalen okkulten Bluttest ab 50 Jahren nach Vollendung des 55. Lebensjahres einer Vorsorge-Darmspiegelung unterziehen, ohne dass bei ihnen ein erhöhtes Risiko oder ein Verdacht besteht, und diese Untersuchung einmal nach 10 Jahren wiederholen lassen.

Da die fachgerecht durchgeführte Koloskopie die zuverlässigste Methode zur Früherkennung und zusätzlich durch Entdeckung und Abtragung größerer Adenome zur Verhinderung von Darmkrebs ist, begrüßen wir diese Änderung nachdrücklich. Ebenso war es an der Zeit, für diese Untersuchung Qualitätsrichtlinien nach dem neuesten Stand zu entwickeln und für diejenigen vorzuschreiben, die die Vorsorge-Darmspiegelung durchführen werden. Wir plädieren nachdrücklich dafür, dass alle Gastroenterologen in diesen Prozess einbezogen werden, unabhängig davon, ob sie in der niedergelassenen Praxis, in niedergelassenen Kompetenzzentren oder in klinischen Schwerpunkteinrichtungen tätig sind. Voraussetzung darf allein die ärztliche Kompetenz sein.

Allerdings wurden im Rahmen dieser Änderungen weitere Regelungen getroffen, die die angestrebte Verbesserung der Früherkennung von Darmkrebs möglicherweise beeinträchtigen bzw. auf den ersten Blick sogar als eine Verschlechterung angesehen werden könnten. In den folgenden Punkten sollen einige der Änderungen der Krebsfrüherkennungsrichtlinien erläutert und auf Verbesserungsbedarf hingewiesen werden:

1. Die Erhöhung des Lebensalters für den Beginn des jährlichen Screenings auf Blut im Stuhl von 45 auf 50 Jahre erscheint eine Verschlechterung der Vorsorge. Diese Vorgehensweise bedeutet jedoch eine Anpassung an nationale und internationale Leitlinien. Es ist $\mathrm{zu}$ bedenken, dass Darmkrebsfälle vor dem 50. Lebensjahr selten auftreten.
Ein erheblicher Anteil dieser frühen Fälle betrifft Personen mit erhöhtem Darmkrebsrisiko wie zum Beispiel erstgradigen Angehörigen von Darmkrebspatienten. Es ist daher wichtig, diese Risikogruppen mittels Familienanamnese zu identifizieren und in gesonderte Überwachungsprogramme aufzunehmen. Die Überwachung beginnt bei Risikopersonen zu einem früheren Zeitpunkt und beinhaltet immer eine Dickdarmspiegelung, ein Screening auf Blut im Stuhl ist nicht ausreichend. Beginn und Durchführung des übrigen bestehenden Krebsvorsorgeprogramms bleiben unverändert und sind weiterhin ein wesentlicher Bestandteil der Bemühungen, die Krebsmortalität zu senken.

2. Hauptvorsorgeuntersuchung ab 55 Jahren sollte die Koloskopie sein. Für diejenigen, die eine Screening-Koloskopie ablehnen, ist die Untersuchung auf Blut im Stuhl ab 55 Jahren dann nur noch alle 2 Jahre, statt wie bisher üblich jährlich, vorgesehen. Ein 2-Jahresabstand für die Untersuchung auf Blut im Stuhl ist jedoch weder praktikabel, noch wissenschaftlich begründet, hier ist eine Beibehaltung der jährlichen Testung zu fordern. Um eine optimale Effektivität des Vorsorgeprogramms zu erreichen, sollte eine wesentliche Aufgabe darin bestehen, auf eine möglichst hohe Teilnahmerate an Koloskopie oder Stuhltestung in der Bevölkerung hinzuwirken.

3. Qualitätsrichtlinien und Hygienemaßstäbe müssen obligater Bestandteil eines jeglichen endoskopischen Vorsorgeprogramms sein, um hierdurch einen gleichbleibend hohen Untersuchungsstandard und maximale Patientensicherheit zu gewährleisten. Kürzlich vorgestellte Untersuchungen haben auf vorhandene Hygienemängel hingewiesen, die abgestellt werden müssen. Um eine optimale Gewährleistung der kassenärztlichen Versorgung zu sichern, erscheint die vorgeschriebene Übergangsfrist von 3 Monaten jedoch sehr knapp bemessen. Hier bedarf es einer kurzfristigen

\begin{tabular}{ll}
\hline KARGER & ( ) 2003 S. Karger GmbH, Freiburg \\
Fax +497614520714 & Accessible online at: \\
$\begin{array}{l}\text { E-mail Information@Karger.de } \\
\text { www.karger.com }\end{array}$ & www.karger.com/cga
\end{tabular}


Abstimmung mit den ärztlichen Fachgesellschaften und Berufsverbänden, wie die neuen Anforderungen an Qualität und Hygiene möglichst schnell und praxisnah umgesetzt werden können.

4. Die Akzeptanz der Koloskopie als Vorsorgeuntersuchung für die breite Bevölkerung hängt wesentlich von der Patientenzufriedenheit mit der Untersuchung ab. Um die Patientenzufriedenheit sicherzustellen, sollte zur Koloskopie eine optimale Begleitmedikation angeboten werden. Die erreichte Patientenzufriedenheit sollte als Bestandteil der Qualitätskontrolle evaluiert und dokumentiert werden. Nicht optimistische Erwartungen, sondern einzig und allein erprobte Modelle können und müssen die Grundlage für Veränderungen sein. Kaum ein anderer Krebs bietet so gute Möglichkeiten wie der Darmkrebs, jährlich viele Tausend Menschenleben in Deutschland zu retten. Das neue Angebot der Vorsorge-Koloskopie zusammen mit einer Umsetzung des erfolgreich erprobten Münchner Modellversuchs zur Darm-
krebs-Früherkennung mit dem Okkultblut-Test ist eine Strategie, deren Erfolg zu belegen und deren Kosten zu verantworten sind. Um ein optimales praktikables Vorsorgeprogramm zu gewährleisten, sollte eine rasche Diskussion über einzelne Nachbesserungen der zum 1. Oktober 2002 eingeführten Regelungen erfolgen.

\section{GASTRO LIGA e.V.}

\section{Classen}

LebensBlicke

Stiftung Früherkennung Darmkrebs

J.F. Riemann

Deutsche Gesellschaft für Verdauungs- und Stoffwechselkrankheiten

W.-H. Schmiegel 